This item was submitted to Loughborough's Research Repository by the author.

Items in Figshare are protected by copyright, with all rights reserved, unless otherwise indicated.

\title{
Closed-loop quasi-orthogonal STBCs and their performance in multipath fading environments and when combined with turbo codes
}

PLEASE CITE THE PUBLISHED VERSION

PUBLISHER

(c) IEEE

VERSION

VoR (Version of Record)

\section{LICENCE}

CC BY-NC-ND 4.0

\section{REPOSITORY RECORD}

Toker, Cenk, Sangarapillai Lambotharan, and Jonathon Chambers. 2019. "Closed-loop Quasi-orthogonal Stbcs and Their Performance in Multipath Fading Environments and When Combined with Turbo Codes". figshare. https://hdl.handle.net/2134/5788. 
This item was submitted to Loughborough's Institutional Repository (https://dspace.lboro.ac.uk/) by the author and is made available under the following Creative Commons Licence conditions.

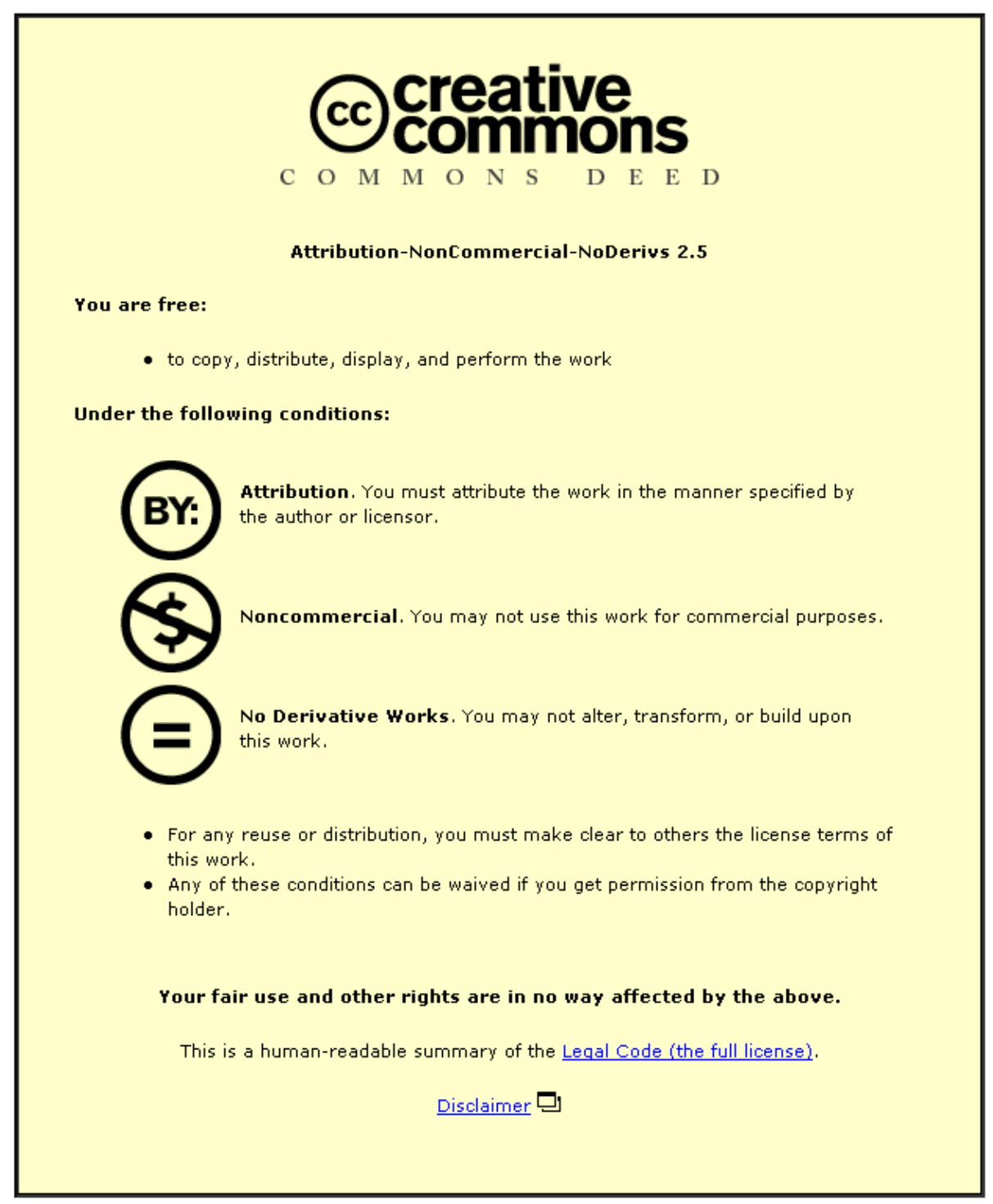

For the full text of this licence, please go to: http://creativecommons.org/licenses/by-nc-nd/2.5/ 


\title{
Closed-Loop Quasi-Orthogonal STBCs and Their Performance in Multipath Fading Environments and When Combined With Turbo Codes
}

\author{
Cenk Toker, Student Member, IEEE, Sangarapillai Lambotharan, Member, IEEE, and \\ Jonathon A. Chambers, Senior Member, IEEE
}

\begin{abstract}
Quasi-orthogonal space-time block codes (QOSTBCs) achieve full code rate at the expense of loss in diversity gain. In this paper, we propose two feedback methods for QO-STBCs to achieve full diversity and full code rate. In the first method, signals radiated from various antennas are rotated by phasors according to feedback from the receiver, whereas the second method is based upon antenna weighting/selection. For high to moderate feedback error rates, it is demonstrated that the proposed methods outperform the quantized transmit beamformer. The performance improvement is also investigated for these closed-loop methods when the transmitted signal is error control coded.
\end{abstract}

Index Terms-Antenna selection, beamformers, closed loop feedback, frequency selective channel, quasi-orthogonal space-time block codes (QO-STBCs), transmit diversity, turbo codes.

\section{INTRODUCTION}

D IVERSITY is an effective method to overcome the destructive effects of fading. In this paper, we will concentrate on two types of transmit diversity: transmit beamforming and space time block codes. Transmit beamforming could enhance the signal quality by the transmitter directing a beam toward the receiver. Full channel state information (CSI), however, is required at the transmitter. If the CSI is not reliable due to feedback errors, the performance of the beamformer could become very poor. Space-time block codes (STBCs) introduced in [2] and [8] are efficient methods to achieve diversity. In [4], quasi-orthogonal STBCs (QO-STBCs) have been introduced as a new family of STBC. These codes achieve full code rate at the expense of reduced diversity. As it will be proved in Section II, the loss of diversity in QO-STBCs is due to a coupling term between the estimated symbols.

In this paper, we propose and investigate two feedback schemes to orthogonalize QO-STBCs by rotating/weighting the transmitted signals from certain antennas in a prescribed way, based upon the information fed back from the receiver [10]. We further show that with only 2-bit feedback as facilitated in UMTS-FDD and for a slow fading channel, full diversity can be achieved. However, there remains a 6-dB gap between the performance of this feedback scheme and the performance of

Manuscript received April 27, 2002; revised September 24, 2003. The editor coordinating the review of this paper and approving it for publication is Y.-C. Liang. This work was supported by the Engineering and Physical Sciences Research Council (EPSRC), U.K., under the Grant GR/S16775/01.

C. Toker and S. Lambotharan are with the Centre for Digital Signal Processing Research, King's College London, WC2R 2LS, U.K. (e-mail: cenk.toker@ieee.org, s.lambotharan@kcl.ac.uk).

J. A. Chambers is with the Centre of Digital Signal Processing, Cardiff School of Engineering, Cardiff, CF24 0YF, Wales, U.K. (ChambersJ@cf.ac.uk).

Digital Object Identifier 10.1109/TWC.2004.837401 a transmit or receive beamformer. In [5] and [11], an antenna weighting algorithm has been proposed for orthogonal STBCs, particularly for Alamouti's block code [2] for two transmit antennas to reduce the performance gap between the beamformer and the STBC. Here, we extend this algorithm to QO-STBC codes and show that the transmit antenna weighting/selection method exploits the structural property of the QO-STBC, which narrows down this performance gap to $2.5 \mathrm{~dB}$, while retaining minimal amount of feedback ( 2 or 3 bits) [10]. It is also shown that the proposed feedback algorithms outperform the performance of quantized transmit beamformers under moderate feedback error rates. The performance is also studied when the proposed method is concatenated with parallel turbo codes.

The organization of this paper is as follows. In Section II, the problem statement is provided. In Sections III and IV, transmit antenna phase rotation and weighting/selection methods based on feedback are introduced. The simplifications for the first method are provided in Section V. In Section VI, the concatenation of the closed-loop methods with parallel turbo codes is explained. Finally, simulation results are presented and conclusions are drawn in Sections VII and VIII, respectively.

\section{Problem Statement}

In [4], a QO-STBC has been proposed with the following code matrix (variations of this method can be found in [6] and [9])

$$
\mathbf{C}=\left[\begin{array}{cccc}
s_{1} & s_{2} & s_{3} & s_{4} \\
-s_{2}^{*} & s_{1}^{*} & -s_{4}^{*} & s_{3}^{*} \\
-s_{3}^{*} & -s_{4}^{*} & s_{1}^{*} & s_{2}^{*} \\
s_{4} & -s_{3} & -s_{2} & s_{1}
\end{array}\right]
$$

This is an extension of Alamouti's $2 \times 2$ scheme [2] to four transmit antennas, where $s_{i}, i=1,2,3,4$ denotes the complex valued transmitted symbols. The operator $(\cdot)^{*}$ denotes complex conjugation. We assume the above code is transmitted through a code division multiple access scheme. The channel is assumed to be frequency selective, and the signal is received with possibly multiple receive antennas. The transmission paths $h_{i, l}$, $i=1, \ldots, 4$, and $l=1, \ldots, L$, are assumed to be resolvable and independent complex valued random variables with zero mean and unity variance, where $i$ denotes the transmit antenna index and $l$ denotes the multipath or receive antenna index. We also assume $h_{i, l}$ remains constant over four symbol intervals. The received signal for each path $l$ at the output of the code matched 
filter (and after complex conjugating the second and third symbols) over four symbol intervals can be written as

$$
\begin{aligned}
{\left[\begin{array}{c}
r_{1, l} \\
r_{2, l}^{*} \\
r_{3, l}^{*} \\
r_{4, l}^{*}
\end{array}\right] } & =\left[\begin{array}{cccc}
h_{1, l} & h_{2, l} & h_{3, l} & h_{4, l} \\
h_{2, l}^{*} & -h_{1, l}^{*} & h_{4, l}^{*} & -h_{3, l}^{*} \\
h_{3, l}^{*} & h_{4, l}^{*} & -h_{1, l}^{*} & -h_{2, l}^{*} \\
h_{4, l} & -h_{3, l} & -h_{2, l} & h_{1, l}
\end{array}\right]\left[\begin{array}{c}
s_{1} \\
s_{2} \\
s_{3} \\
s_{4}
\end{array}\right]+
\end{aligned}
$$

where $n_{i, l}, i=1,2,3,4$ are the zero-mean, circularly symmetric, complex valued Gaussian noise components with two sided power spectral density $N_{o} / 2$ per dimension. The power of the transmitted signals at each antenna is scaled so that the total output power of the four antennas is unity. Stacking $\mathbf{r}_{l}$, and $\mathbf{n}_{l}$ in a vector and $\mathbf{H}_{l}$ into a tall matrix, $\left(\mathbf{r}=\left[\begin{array}{llll}\mathbf{r}_{0}^{T} & \mathbf{r}_{1}^{T} & \ldots & \mathbf{r}_{L-1}^{T}\end{array}\right]^{T}\right.$, $\left.\mathbf{H}=\left[\begin{array}{llll}\mathbf{H}_{0}^{T} \mathbf{H}_{1}^{T} & \ldots & \mathbf{H}_{L-1}^{T}\end{array}\right]^{T}, \mathbf{n}=\left[\begin{array}{llll}\mathbf{n}_{0}^{T} & \mathbf{n}_{1}^{T} & \ldots & \mathbf{n}_{L-1}^{T}\end{array}\right]^{T}\right)$, and applying channel matched filtering, [6], we obtain

$$
\mathbf{r}_{m f}=\mathbf{H}^{H} \mathbf{r}=\Delta \mathbf{s}+\mathbf{n}
$$

where $(\cdot)^{H}$ denotes Hermitian transpose. The matrix $\Delta=$ $\mathbf{H}^{H} \mathbf{H}$ is a $4 \times 4$ matrix with entries $\gamma=\sum_{l=0}^{L-1} \sum_{i=1}^{4}\left|h_{i, l}\right|^{2}$, and $\alpha=\sum_{l=0}^{L-1} \operatorname{Re}\left\{h_{1, l}^{*} h_{4, l}-h_{2, l}^{*} h_{3, l}\right\}$ as follows:

$$
\boldsymbol{\Delta}=\left[\begin{array}{cccc}
\gamma & 0 & 0 & \alpha \\
0 & \gamma & -\alpha & 0 \\
0 & -\alpha & \gamma & 0 \\
\alpha & 0 & 0 & \gamma
\end{array}\right]
$$

For an orthogonal STBC, all the off-diagonal terms of $\Delta$ will be zero as in Alamouti's scheme, whereas for QO-STBCs, some nonzero off-diaogonal terms appear, yielding coupling between estimated symbols, therefore, reducing the diversity gain of the code. Our aim is to eliminate or reduce the off-diagonal element. In the cases when this term cannot be made zero, an equalizer should be employed to improve performance. It can be shown that the SNR at the receiver is

$$
\mathrm{SNR}_{\mathrm{zf}}=\frac{\gamma^{2}-\alpha^{2}}{\gamma} \frac{\sigma_{\mathrm{s}}^{2}}{\sigma_{\mathrm{n}}^{2}}
$$

where $\sigma_{s}^{2}$ is the total power of the transmitted signal at the receive antenna, which is equal to unity in our case, and $\sigma_{n}^{2}$ is the noise power at the receiver. Therefore, minimizing the off-diagonal term $\alpha$ maximizes the signal to noise ratio. The value of $\alpha$ can be reduced to zero by rotating or weighting the transmitted signal as explained in the following sections.

\section{ORthogonalization by Phase Rotation}

Consider that the signals from the third and fourth transmit antennas are respectively rotated by two phasors, $\phi$ and $\theta$, then the term $\alpha$ in (4) can be written as

$$
\begin{aligned}
\alpha^{\prime} & =\operatorname{Re}\left\{\sum_{l=0}^{L-1} h_{1, l}^{*} h_{4, l} e^{j \theta}-\sum_{l=0}^{L-1} h_{2, l}^{*} h_{3, l} e^{j \phi}\right\} \\
& =|\kappa| \cos (\theta+\angle \kappa)-|\lambda| \cos (\phi+\angle \lambda)
\end{aligned}
$$

where $\kappa=\sum_{l=0}^{L-1} h_{1, l}^{*} h_{4, l}$ and $\lambda=\sum_{l=0}^{L-1} h_{2, l}^{*} h_{3, l}$. Here, | . I and $\angle$ denote, respectively, the absolute value and the angle (arctan) operators. Clearly, this would not change the transmit power. After trigonometric manipulations, it can be shown that $\left(\alpha^{\prime}=0\right)$ has infinitely many solutions for $\theta$ and $\phi$. By defining $\xi=\arccos (|\kappa| /|\lambda|)$, the solution can be written as

$$
\theta=\arccos \left(\frac{|\lambda|}{|\kappa|} \cos (\phi+\angle \lambda)\right)-\angle \kappa
$$

provided that $\phi$ is in the range $\phi \in[0,2 \pi)$ if $|\lambda|<|\kappa|$, or otherwise, $\phi \in[\pi-\xi-\angle \lambda, \xi-\angle \lambda] \cup[-\xi-\angle \lambda, \pi+\xi-\angle \lambda]$.

\section{ORthogonalization by ANTENNA SElECtion}

We extend the method proposed in [5], which is for two antennas to QO-STBC by multiplying the transmitted signal by a diagonal weighting matrix $\mathbf{W}$, which is drawn from the set $\Omega=\left\{\mathbf{W}_{1}, \mathbf{W}_{2}, \mathbf{W}_{3}, \mathbf{W}_{4}\right\}=$ $\left\{\operatorname{diag}\left\{|\mathrm{a}|^{2},|\mathrm{a}|^{2}, 1-|\mathrm{a}|^{2}, 1-|\mathrm{a}|^{2}\right\}^{1 / 2}, \operatorname{diag}\left\{|\mathrm{a}|^{2}, 1-\right.\right.$ $\left.|\mathrm{a}|^{2},|\mathrm{a}|^{2}, 1-|\mathrm{a}|^{2}\right\}^{1 / 2}, \operatorname{diag}\left\{1-|\mathrm{a}|^{2},|\mathrm{a}|^{2}, 1 \quad-\right.$ $\left.\left.|a|^{2},|a|^{2}\right\}^{1 / 2}, \operatorname{diag}\left\{1-|a|^{2}, 1-|a|^{2},|a|^{2},|a|^{2}\right\}^{1 / 2}\right\}$, where $\operatorname{diag}\{\cdot\}$ is a diagonal matrix with all zero entries except the main diagonal and $|a|^{2} \leq 1$. The case $|a|^{2}=0.5$ corresponds to no selection and all antennas transmit at the same power level. At the receiver, the quality of the channels is measured in terms of the norms of the individual channels, and the appropriate weighting matrix is chosen by exploitation of the 2-bit information sent to the transmitter by feedback. For example, if $\sum_{l}\left|h_{1, l}\right| \geq \sum_{l}\left|h_{4, l}\right|$ and $\sum_{l}\left|h_{2, l}\right| \geq \sum_{l}\left|h_{3, l}\right|$, the matrix $\mathbf{W}_{1}$ is chosen.

The performance can be further improved if the rows of $\mathbf{C}$ can be permuted. According to this setup, if $|a|=1$, only four combinations of antennas (Ant 1, Ant 2), (Ant 1, Ant 3), (Ant 4, Ant 2), (Ant 4, Ant 3) are chosen. Suppose the signals received from the second and third antennas are stronger than the others, we should choose (Ant 2, Ant 3). Permutation of $\mathbf{C}$ allows this but requires one more bit of feedback.

\section{REDUCTION OF FEEDBACK}

Due to practical limitations, the number of feedback bits required from the receiver to the transmitter should be kept as small as possible. In the sequel, we provide two methods to reduce the number of feedback bits while retaining satisfactory forward link performance.

\section{A. Single Phase Feedback}

Instead of rotating the signal from both antennas, only one of them can be rotated. With rotation applied only at the fourth antenna, for example, $\alpha$ can be rewritten as

$$
\begin{aligned}
\alpha^{\prime \prime} & =\operatorname{Re}\left\{\sum_{l=1}^{L} h_{1, l}^{*} h_{4, l} e^{j \theta}-\sum_{l=1}^{L} h_{2, l}^{*} h_{3, l}\right\} \\
& =|\kappa| \cos (\theta+\angle \kappa)-\lambda_{r}
\end{aligned}
$$

where $\lambda_{r}$ is the real part of $\lambda$. Under the condition $\left|\lambda_{r}\right| \leq|\kappa|$, $\alpha^{\prime \prime}=0$ has two solutions for $\theta$

$$
\theta i=(-1)^{i} \arccos \left(\frac{\lambda_{r}}{|\kappa|}\right)-\angle \kappa, \quad i=1,2 .
$$




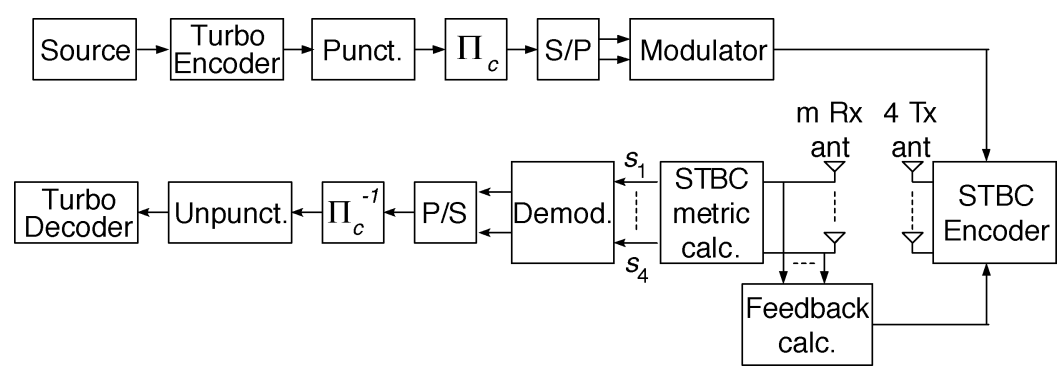

Fig. 1. Transmitter and receiver structures of the proposed scheme for closed loop STBC, together with a turbo encoder interconnected with a bit interleaver and puncturing operation.

On the other hand, if $\left|\lambda_{r}\right|>|\kappa|$, there is no solution for $\alpha^{\prime \prime}=0$ and $\left|\alpha^{\prime \prime}\right|$ can only be minimized at the phase angle

$$
\theta= \begin{cases}\pi-\angle \kappa, & \text { if }|\kappa|<-\lambda_{r} \\ -\pi-\angle \kappa, & \text { if }|\kappa|<\lambda_{r}\end{cases}
$$

\section{B. Quantization}

Equations (10) and (11) for the single phase and (8) for the two phase cases provide optimal phase angles with infinite precision which would require a large feedback overhead to express the floating/fixed point values of the phases. Assuming a practical scenario that only $K$ bits are allowed for the feedback, then for the single antenna phase adjustment, the discrete feedback information corresponding to the phase $\theta$ will be an element of the set $\left\{\tilde{\theta} \in \Omega=\left\{2 \pi k / 2^{K}\right\}, k=0,1, \ldots, 2^{K}-1\right\}$ computed as

$$
\tilde{\theta}=\arg \min _{\tilde{\theta} \in \Omega} \alpha^{\prime \prime}(\tilde{\theta}) .
$$

Similarly, for the dual antenna phase adjustment, the discrete estimated feedback information for the phases $\theta$ and $\phi$ are the elements of the set $\left\{\{\tilde{\theta}, \tilde{\phi}\} \in \Omega=\left\{\left(2 \pi k / 2^{(K-1)}\right)\right\}, k=\right.$ $\left.0,1, \ldots, 2^{(K-1)}-1\right\}$ computed as

$$
\{\tilde{\theta}, \tilde{\phi}\}=\arg \min _{\{\tilde{\theta}, \tilde{\phi}\} \in \Omega} \alpha^{\prime}(\tilde{\theta}, \tilde{\phi}) .
$$

\section{Concatenation With Turbo Codes}

In many wireless applications, it is favorable to have an outer error correction code to correct the errors that remain after inner codes. In this section, concatenation of the proposed scheme with parallel turbo codes is investigated, as in Fig. 1.

Consider that a binary data sequence of length $L_{u}$ is to be transmitted. First, it is encoded by the binary turbo encoder where a random interleaver is used, following which, to increase the bit rate puncturing may be applied, [3]. A bit interleaver is employed before modulation to avoid correlation between the codeword bits corresponding to the same information bit by being positioned in the same channel symbol. After the interleaver, modulation is performed by packing the incoming bits into groups with appropriate size and the resulting symbols are transmitted according to the STBC described in Section II.

It is assumed that the channel between each transmit and receive antenna experiences independent fading. At the receiver, the "soft" values of the symbols of an STBC codeword are taken as the output of the matched filter, given in (3). The matrix $\Delta$ is assumed to be diagonal with all off-diagonal terms equal to zero, since the coupling term is made zero by the feedback connection, and the resulting soft estimates of the symbols are assumed to be uncoupled. These soft estimates are appended to construct the whole transmission frame which will be parallel-to-serial converted and bit deinterleaved before turbo decoding.

The operation of the turbo decoder can be found in many sources (i.e., [3]), therefore, it is not going to be explained here in detail. However, it is useful to note that the MAP metric calculations differ from the standard Rayleigh fading channel derivations. The metrics can be calculated from

$$
P\left\{\mathbf{y}_{k} \mid u_{k}, \boldsymbol{\gamma}_{k}\right\}=M \exp \left\{\frac{1}{2} L_{c} y_{k}^{s} u_{k}^{s}+\frac{1}{2} L_{c} y_{k}^{p} u_{k}^{p}\right\}
$$

where $M$ includes constants and terms independent of the information bit, $u_{k}$. We define the received codeword bits and channel gain corresponding to the $k$ th information bit, $u_{k}$, respectively, as $\mathbf{y}_{k}=\left[y_{k}^{s} y_{k}^{p}\right]^{T}$ and $\gamma_{k}=\left[\gamma_{k}^{s} \gamma_{k}^{p}\right]^{T}$. The term $y_{k}^{s}$ is the received systematic soft bit and $y_{k}^{p}$ is the corresponding received parity soft bit of $u_{k}$, and $u_{k}^{p}$ is the corresponding parity bit after encoding. The term $\varepsilon_{s}$ is the encoded bit energy in the channel, and $L_{c}=2 \varepsilon_{s} / N_{o}$.

\section{Simulations AND Results}

For the simulations, a UMTS FDD model [1] is assumed. A high data rate service with a spreading factor of 8 has been chosen. Four (eight) uncorrelated fading waveforms are generated to model the single (multi)-path channel from each transmit antenna to the receive antenna.

In Fig. 2, we depict the BER comparison of the open loop QO-STBC, the two proposed feedback schemes (phase feedback and antenna selection), together with the performance of a transmit beamformer, all for four transmit and one receive antennas over flat-fading channels. The transmit beamformer is realized by multiplying each antenna by the complex conjugate of the corresponding channel coefficient and normalizing to have unity total transmit power. Two cases for the transmit beamformer are considered. First, all four transmit antennas are used, and second, for comparison purposes the number of transmit antennas is decreased to two by selecting the best two antennas out of four. Also, it should be noted that we used full CSI instead of quantized CSI for the transmit beamformers as a benchmark. But degradation in performance should be expected for the beamformer with quantization. For the antenna 


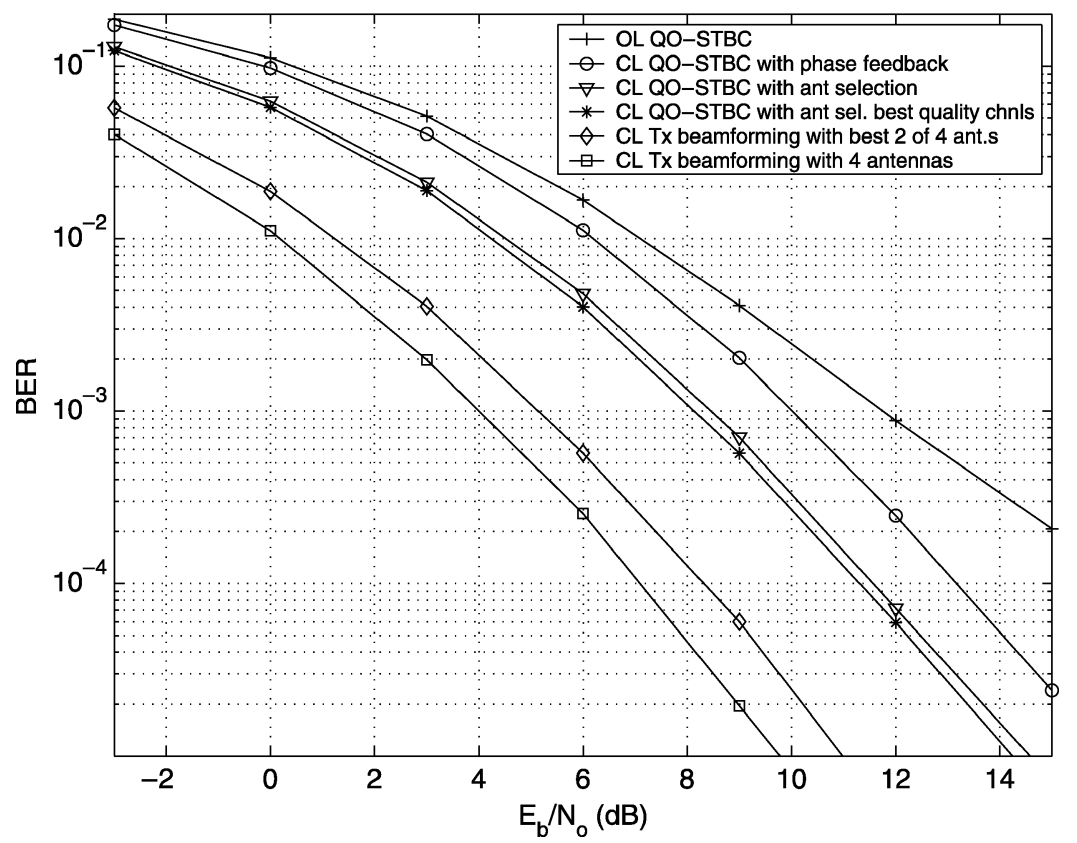

Fig. 2. Bit-error-rate performance comparison of the open loop quasi-orthogonal code, the closed loop transmit beamformer with two and four antennas, and the proposed phase feedback and the antenna selection feedback (i.e., $|a|^{2}=1$ ) schemes, $\mathrm{Pe}=0.001$.

weighting/selection algorithm, the case $|a|^{2}=1$ is investigated, and in this case, there is no need for a phase feedback, as $\alpha=0$. Again, two cases are examined for this scheme; the first one is to switch off the two transmit antennas by choosing $\mathbf{W}$ from $\Omega$, and the second one is to permute the rows of $\mathbf{C}$ and then choosing $\mathbf{W}$ from $\Omega$. While the first method requires two bit feedback, the second method needs three bits to indicate the best possible antenna combinations. The difference in performance between the two antenna transmit beamformer and the three bit feedback QO-STBC is approximately $2.8 \mathrm{~dB}$ at bit-error rate (BER) of $10^{-3}$. In fact, the performance gain between the three bit feedback and the two bit feedback is only $0.2 \mathrm{~dB}$, which is not very significant considering the feedback overhead involved. The performance of QO-STBC with antenna selection is $5 \mathrm{~dB}$ better than that without feedback. Therefore, the performance gap between the QO-STBC and the transmit beamformer is reduced from $6.5 \mathrm{~dB}$ to $2.5 \mathrm{~dB}$ at $\mathrm{BER}=10^{-3}$ when feedback is employed. Two phase feedback also introduces a gain of approximately $2 \mathrm{~dB}$ at $\mathrm{BER}=10^{-3}$, with respect to the open-loop curve which is minor compared to antenna selection, but the robustness of phase feedback schemes will be demonstrated in the next set of simulations.

In Fig. 3, we provide comparison of the proposed closed loop feedback schemes with a quantized transmit beamformer for various values of the feedback error rate, $P_{e}$. The transmit beamformer is realized as follows; the four transmit antennas are multiplied by the vector $\left[1 e^{j \theta_{1}} e^{j \theta_{2}} e^{j \theta_{3}}\right]^{T}$ where $\left\{\theta_{1}, \theta_{2}, \theta_{3}\right\} \in\{0, \pi\}$. Therefore, each phase value is determined by one bit, which is fed back from the receiver to maximize the SNR at the receiver. For the closed loop antenna selection method, two bits are used as discussed in Section IV, and for the closed loop phase feedback scheme, a single phase with two bit feedback is used as explained in Section V. In Fig. 3, the dark shaded region represents the span of the BER performance of the phase feedback method for two extreme levels of feedback bit error rates, $P_{e}=0$ and $P_{e}=0.5$. The light shaded region is the span of the BER performance for the antenna selection based closed-loop method. The BER performance curves for various $P_{e}$ values ranging from 0 to 0.5 are also provided for the quantized transmit beamformer. Moderate feedback error for transmit diversity schemes is possible even for a high quality uplink channel due to the inability of the closed loop mechanism to track the variation of the downlink channel (i.e., the propagation environment could have changed by the time the transmitter uses the feedback information for precoding). In the worst case that corresponds to $P_{e}=0.5$, the transmit beamformer performance is equivalent to the single antenna Rayleigh fading performance. For the phase feedback based QO-STBC scheme, the worst case performance is equivalent to open loop QO-STBC, which is also the same as two antenna STBC, which outperforms the single antenna Rayleigh fading performance. In the worst case scenario, the performance of the antenna selection method is slightly worse than the phase rotation method, but still significantly better than that of the transmit beamformer. From Fig. 3, it can be deduced that both proposed closed loop schemes outperform the transmit beamformer for a reasonable range of the feedback BERs, especially at high SNR, demonstrating that the closed loop STBC algorithms are robust to a highly dynamic mobile environment.

In Fig. 4, we investigate the BER performance of the proposed closed loop phase feedback STBC for a frequency selective fading channel with two equal power paths. The performance is compared with one transmit antenna and a four receive antenna maximal ratio receive combiner (MRRC). Apart from a $6 \mathrm{~dB}$ gain, the BER curves of the closed loop STBC and the MRRC appear to be identical. The $6 \mathrm{~dB}$ difference in gain is due to the fact that for STBC, the total transmit power for both the 


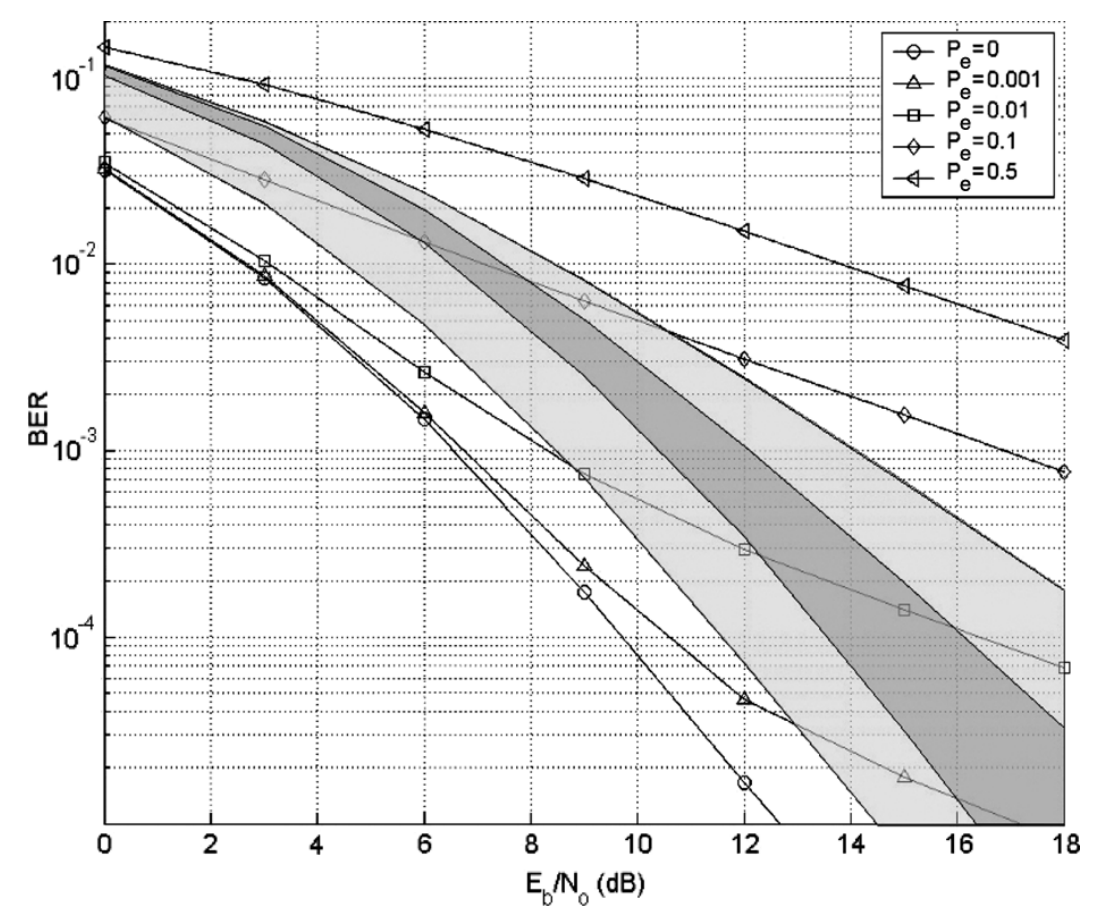

Fig. 3. Bit-error-rate performance comparison of the proposed closed loop schemes and 3-bit quantized transmit beamformer for various values of the feedback error rate, $P_{e}$, the light shaded area corresponds to the closed loop antenna selection method (2-bit), and the dark shaded area corresponds to the closed loop 2-bit quantized single phase feedback.

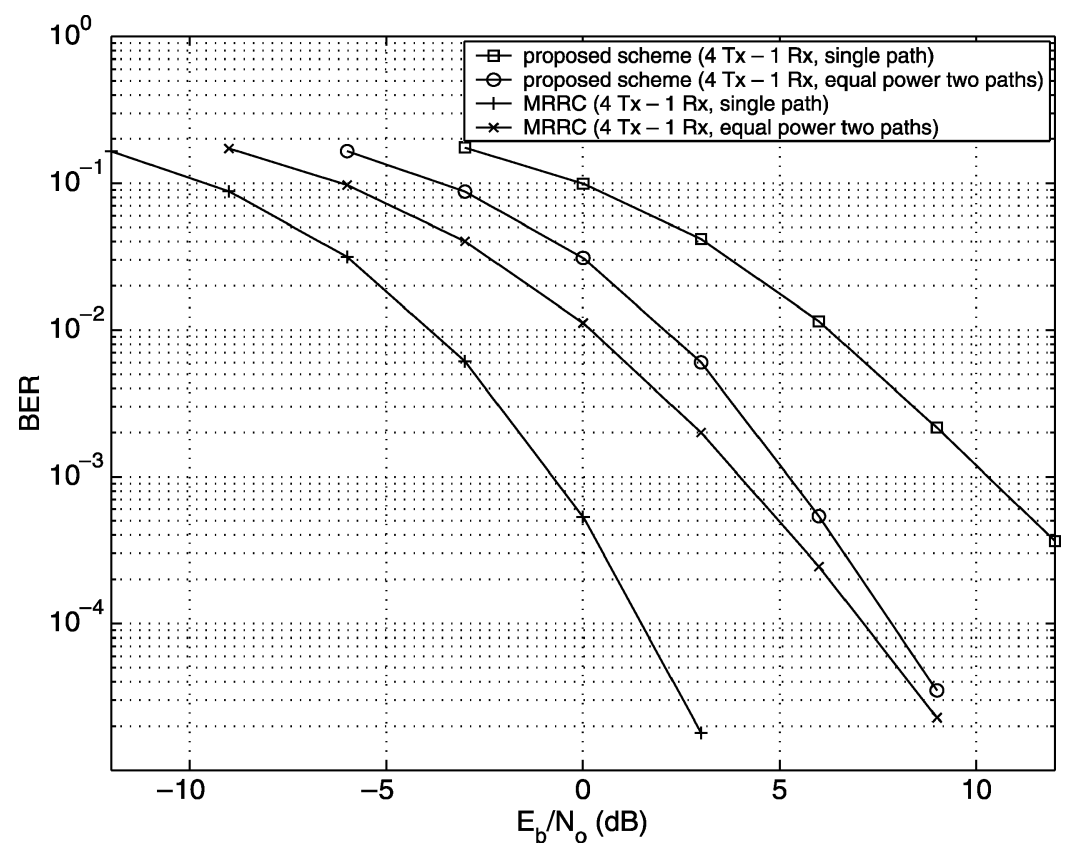

Fig. 4. Bit-error-rate comparison of the proposed closed loop four transmit one receive antenna scheme with both single and multipath (two equal power paths) channels having two infinite precision phase feedbacks and the one transmit four receive antenna maximal ratio combiner over both single and multipath channels for a UMTS FDD model at mobile speed $3 \mathrm{~km} / \mathrm{h}$.

STBC and MRRC schemes is kept the same, i.e., the transmit power per antenna for STBC is a quarter of the total transmit power. In Fig. 4, the BER performances of STBC and MRRC for flat fading channel are also given for comparison.

For the final set of simulations, in Figs. 5 and 6, we provide the performance when the proposed methods are concatenated with parallel turbo codes, for both punctured (rate 1/2) and unpunctured (rate 1/3) scenarios. The generating function for each constituent code is $(7,5)_{8}$. For the unpunctured codes, the proposed methods outperform the open-loop QO-STBC with $1 \mathrm{~dB}$ for the phase feedback method, and approximately 2.75 $\mathrm{dB}$ for the antenna selection method at BER $=10^{-3}$ after 16 iterations. For punctured codes, the performance gap between the antenna selection and open-loop QO-STBC is approximately $3.4 \mathrm{~dB}$, while the gap between the phase feedback method and open-loop QO-STBC is $1.5 \mathrm{~dB}$ at $\mathrm{BER}=10^{-3}$. 


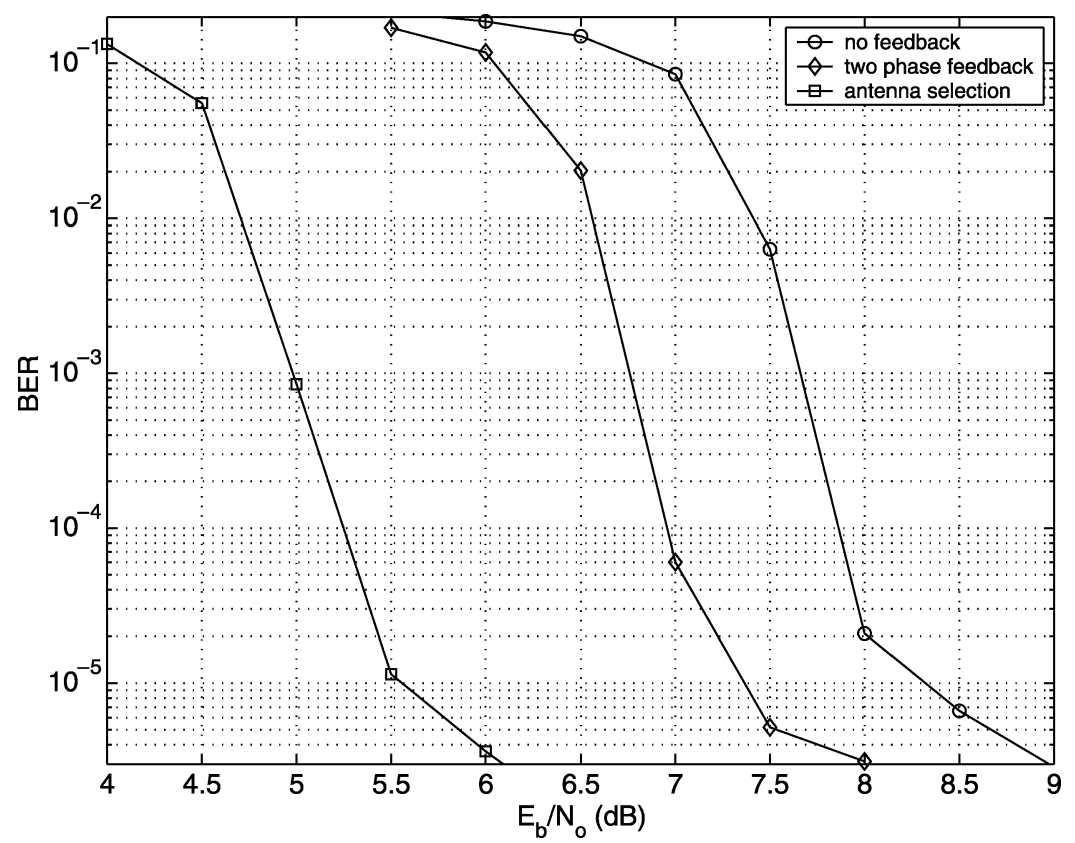

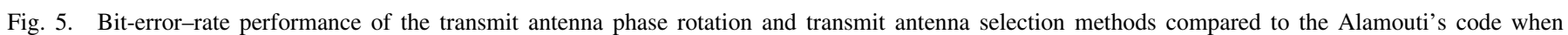
concatenated with parallel turbo code without puncturing after 16 iterations.

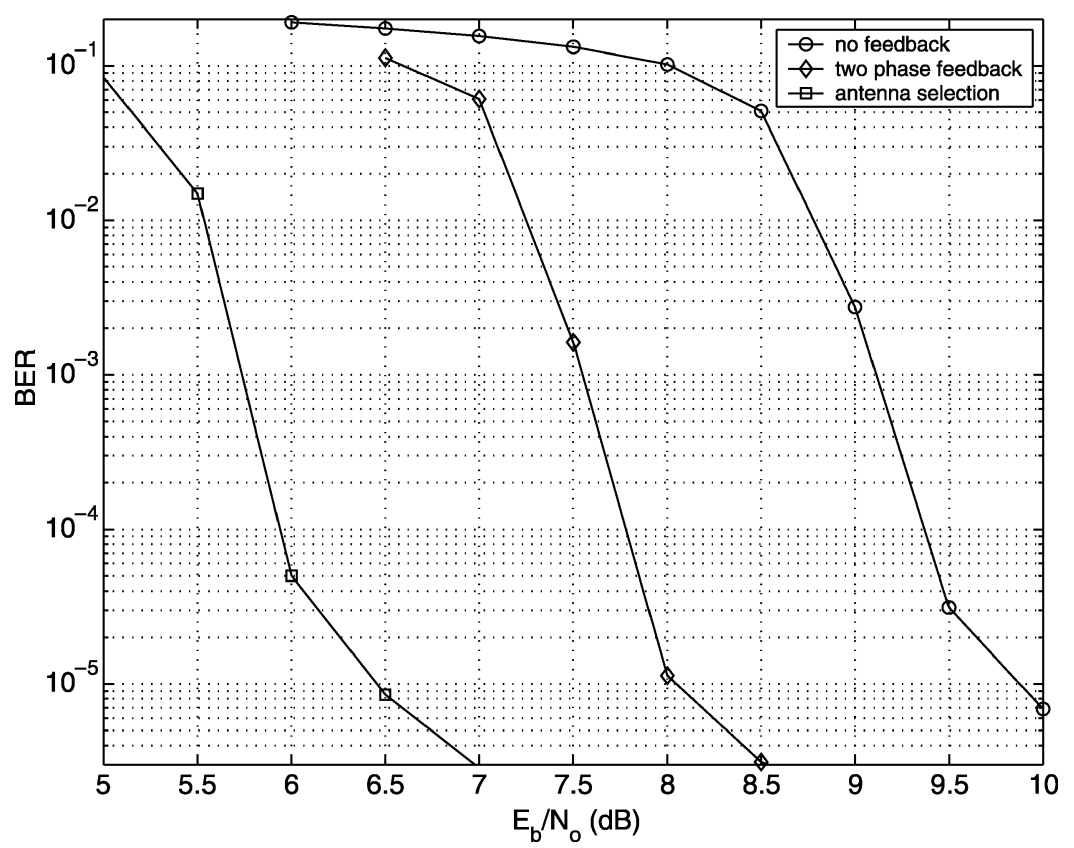

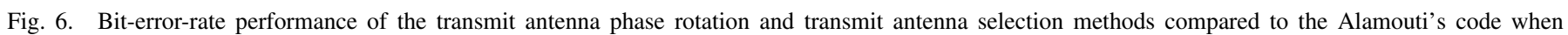
concatenated with parallel turbo code with puncturing after 16 iterations.

\section{CONCLUSION}

In this paper, we proposed two closed loop feedback methods for QO-STBCs and investigated their performance under single and multipath fading scenarios with and without error control codes. Closed-loop methods based upon phase feedback and antenna selection have been investigated. It is shown that the performance gap between the uncoded QO-STBC and the four antenna transmit beamformer is reduced to $2.5 \mathrm{~dB}$ from $6.5 \mathrm{~dB}$ using feedback. It is also shown that the proposed methods outperform a three-bit quantized transmit beamformer at moderate to high-feedback error rates, resulting in the proposed schemes being more robust to the dynamics in the mobile environment. To include a more practical scenario, we studied the effect of quantization and reduced the number of phases, and found that single phase feedback with 2-bit resolution produces substantial performance gain. Investigation of multipath fading revealed that each independent path increases the system performance and order of diversity, and two bit feedback is still adequate. Finally, for the concatenation with parallel turbo codes, a performance gain of approximately 2.75 $\mathrm{dB}$ is observed for the unpunctured case, and approximately, 
a $3.4 \mathrm{~dB}$ gain is achieved for the punctured case as compared to the open-loop QO-STBC.

\section{ACKNOWLEDGMENT}

The authors would like to acknowledge the anonymous reviewers for their comments and their reference to the independent work of Roquette et al. [7], during the review process.

\section{REFERENCES}

[1] 3GPP TS 25.211, Physical Channels and Mapping of Transport Channels Onto Physical Channel (FDD), Rel. 5, 2002.

[2] S. M. Alamouti, "A simple transmit diversity technique for wireless communications," IEEE J. Select. Areas Commun., vol. 16, pp. 1451-1458, Oct. 1998.

[3] E. K. Hall, "Performance and design of turbo codes on Rayleigh Fading Channels," M.Sc. dissertation, Dept. Elect. Eng., Univ. Virginia, Charlottesville, 1996.

[4] H. Jafarkhani, "A quasi-orthogonal space-time block code," IEEE Trans. Commun., vol. 49, pp. 1-4, Jan. 2001.
[5] E. G. Larsson, G. Ganesan, P. Stoica, and W. H. Wong, "On the performance of orthogonal space-time block coding with quantized feedback," IEEE Commun. Lett., vol. 6, pp. 487-489, Nov. 2002.

[6] C. B. Papadias and G. J. Foschini, "A space-time coding approach for systems employing four transmit antennas," Proc. IEEE Int. Conf. Acoust., Speech, Signal Processing, pp. 2481-2484, May 2001.

[7] S. Rouquette, S. Merigeault, and K. Gosse, "Orthogonal full diversity space-time block coding based on transmit channel state information for 4 Tx antennas," Proc. IEEE Int. Conf. Commun., vol. 1, pp. 558-562, May 2002.

[8] V. Tarokh, H. Jafarkhani, and A. R. Calderbank, "Space-time block codes from orthogonal designs," IEEE Trans. Inf. Theory, vol. 45, pp. 1456-1467, July 1999.

[9] O. Tirkkonen, A. Boariu, and A. Hottinen, "Minimal nonorthogonality rate 1 space-time block code for 3 + Tx," IEEE ISSSTA, pp. 429-432, Sept. 2000.

[10] C. Toker, S. Lambotharan, and J. A. Chambers, "Space-time block coding for four transmit antennas with closed loop feedback over frequency selective fading channels," in IEEE Inf. Theory Workshop, Paris, France, Mar. 2003, pp. 195-198.

[11] M. Katz, E. Tiirola, and J. Ylitalo, "Combining space-time block coding with diversity antenna selection for improved downlink performance," in IEEE VTC Fall'01, vol. 1, Atlantic City, NJ, Oct. 2001, pp. 178-182. 